\title{
Muon and muon-induced phosphorescence events in the COSINE-100 dark matter searches
}

\author{
Hafizh Prihtiadi* \\ on behalf of the COSINE-100 Experiment \\ Department of Physics, Bandung Institute of Technology, Bandung 40132, Indonesia \\ E-mail: hafizh.prihtiadiegmail.com
}

The COSINE-100 experiment is joint experiment by the KIMS-NaI (Korean Invisible Mass Search) and the DM-Ice which aim to confirm or refute the annual modulation signal reported by DAMA/LIBRA using the same target material. In order to tag and suppress cosmic-ray muons, a muon detector was constructed using plastic scintillator panels that completely surround the crystal detector array at Yangyang Underground Laboratory (Y2L). High energy muons in the $\mathrm{NaI}(\mathrm{Tl})$ crystals and low energy scintillation signals corresponding to muon-induced phosphorescence events with half-lives longer than a few seconds were observed. The muon underground flux was measured to be $328 \pm 1$ (stat.) \pm 10 (syst.) muons $/ \mathrm{m}^{2} /$ day. In this report, a study of muon-induced phosphorescence events in the COSINE-100 are reported.

The 39th International Conference on High Energy Physics (ICHEP2018)

4-11 July, 2018

Seoul, Korea

${ }^{*}$ Speaker. 


\section{Introduction}

COSINE-100 has installed $106 \mathrm{~kg}$ low background of crystal NaI(Tl) for direct search dark matter experiment that immersed by $\sim 2$ tons of liquid scintillators [1, 2]. COSINE-100 has also constructed the muon detector to tag and study the muon-induced phosphorescence events [3]. In ongoing analysis from the physics data, COSINE-100 has observed the phosphorescence events after following of a muon event from $\sim 59$ days accumulated data. Phosphorescence events are primarily at low energy events and comprised of thousands of photons. These events have potential to induce a low energy modulation following the phase of muon modulation. A muon in crystal events has approximately $\sim 1 \mu /$ crystal/hour.

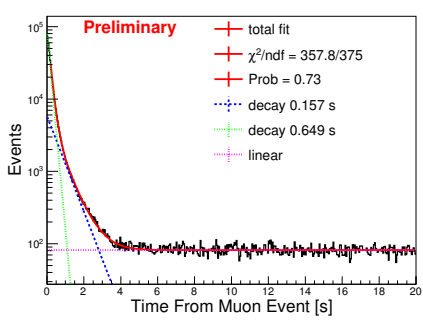

(a)

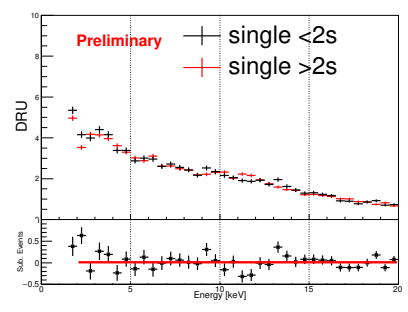

(b)

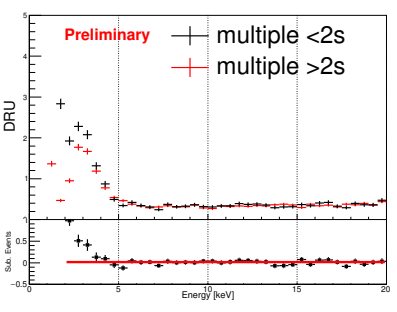

(c)

Figure 1: (a) Muon-induced phosphorescence is observed at crystal 6 of COSINE-100 detector, (b) A single hit spectrum of events in crystal 4, (c) A multiple hit spectrum of events in crystal 4.

In Fig. 1(a), the photons rate increase immediately following the muon events and decays exponentially. Phosphorescence induced by muons has been proposed as a potential modulating background in the region of interest for $\mathrm{NaI}(\mathrm{Tl})$ dark matter experiments. In Fig. 1(b) and (c), this study has selected events below 2 seconds after direct muon and compared with events after 2 seconds. Considering, most of phosphorescence-events are trapped and not rejected by noise removal cut. A Subtracted events of below and after 2 seconds shows there are events in 2-6 keV in the region of interest. This must be considered for the feasibility of muons and muon-induced phosphorescence to mimic a low energy scintillation-like signal.

\section{Summary}

The COSINE-100 experiment has started physics run started September 2016. Muons are identified by their high energy and coincidence requirements. An average observed flux of muons in crystal to be $\sim 1 \mu /$ crystal/hour. In order to understand the phosphorescence events to be the sole source of DAMA signals, the events in the region of interest must be entirely muon-induced, and this is highly improbable.

\section{References}

[1] G. Adhikari et al., Eur. Phys. J. C 78 (2018) 107.

[2] G. Adhikari et al., JINST 13 (2018) P09006.

[3] H. Prihtiadi et al., JINST 13 (2018) T02007. 